\title{
Evaluation of Intra-Operative Abdominal Wall Perfusion in Post-Bariatric Abdominal Dermolipectomy
}

\author{
Adrian Dragu $^{a}$ Stefan Schnürer ${ }^{a}$ Thomas Horbach $^{b} \quad$ Frank Unglaub $^{a}$ \\ Andreas Arkudas ${ }^{a} \quad$ Justus P. Beier ${ }^{a} \quad$ Ulrich Kneser ${ }^{a}$ Raymund E. Horch ${ }^{a}$ \\ a Department of Plastic and Hand Surgery, University Hospital, Friedrich-Alexander-University \\ of Erlangen-Nürnberg, Erlangen, ${ }^{b}$ Department of Surgery, Municipal Hospital Schwabach, \\ Schwabach, Germany
}

\section{Key Words}

Adipose tissue $\cdot$ Bariatric surgery $\cdot$ BMI $\cdot$ Interdisciplinary $\cdot$ Microvasculature $\cdot$ Risk factors Surgery · Dermolipectomy · Post-bariatric plastic surgery $\cdot$ Abdominal wall perfusion • Tissue ischaemia

\begin{abstract}
Objective: Abdominal dermolipectomy after massive weight loss has become a standard procedure. However the complication rates such as wound necrosis or secondary healing complications are still high. In this context ischaemia or inadequate micro-perfusion are known as triggers of wound healing complications. Little is known about the regional perfusion patterns before and after post-bariatric abdominal dermolipectomy. This study focuses on assessment of intraoperative micro-perfusion patterns of the abdominal tissue. Methods: The perfusion of the abdominal wall flap was monitored intra-operatively in 17 patients with an average BMI of $29.2 \pm 3.7 \mathrm{~kg} / \mathrm{m}^{2}$ after bariatric surgery. All patients underwent abdominal post-bariatric dermolipectomy after massive weight loss while applying the non-invasive O2C laser-spectrophotometer. The micro-perfusion parameters oxygen saturation $\left(\mathrm{SO}_{2}\right)$, relative haemoglobin content $(\mathrm{rHB})$ and relative blood flow (BF) were intra-operatively measured. Results: The results of this study show that the part of the abdominal fat typically resected during dermolipectomy has the lowest $\mathrm{SO}_{2}$ before surgery. Furthermore, the results demonstrate that previously well oxygenated parts in the median line of the abdominal fat undergo a significant decrease in oxygen saturation upon mobilisation and subsequent suturing, while the caudal wound edges show an increase of micro-perfusion parameters. Conclusion: Data show that micro-perfusion is worst in the median line of the cranial wound edge and is significantly altered after mobilisation. In addition an intra-operative increase of micro-perfusion in the caudal part of the wound edge, especially in the mons pubis area, can be measured.
\end{abstract}




\section{Introduction}

The technique of abdominoplasty is older than 100 years and has been subjected to continuous advancements - especially in the last 50 years [1-3]. Different incisions such as the bikini line incision have been chosen for cosmetic reasons on the one hand, but also for anatomic and physiologic reasons on the other hand [4,5]. All these improvements helped to make abdominoplasty and post-bariatric abdominal dermolipectomy one of the most common techniques applied today in plastic surgery. According to the 2009 statistics of the American Society for Aesthetic Plastic Surgery on cosmetic surgery, 127,000 abdominoplasties have been performed in the USA in 2009. This represents an overall increase of $276 \%$ compared to 1997 [6].

Despite all improvements in the surgical technique, the complication rates still remain considerable [7]. These complication rates even increase, when obese patients or patients after massive weight loss are treated. In a study on 206 patients, Neaman et al. [8] reported an overall complication rate of $37.4 \%$ with a significant increased complication rate in obese patients. However, obese and overweight patients represent a rapidly growing group of future patients that will undergo abdominal dermolipectomy because their proportion in the population of western countries is rapidly increasing [9]. For patients who have achieved massive weight loss due to bariatric surgery, abdominal dermolipectomy represents the best approach to accomplish and optimise the results [10]. These facts show why it is a major task in post-bariatric plastic surgery to reduce complication rates of abdominal dermolipectomy. Many complications like epidermolysis, seroma formation, wound dehiscence, cellulites or abscess formation and wound healing problems are located in the region of the incision. These may be due to the operative procedure itself, which often results in inadequate blood and oxygen supply of the abdominal flap. According to Mayr et al. [11], the cause of this inadequate blood supply is the anatomy of the vascular territories of the abdominal wall region. Huger et al. [12] therefore proposed a subdivision of the abdominal wall into three different vascular territories. Thereby, the most vulnerable region seems to be the medial cranial part of the resection zone, which is usually supplied by the inferior and superior epi-gastric artery. This region loses the supply of the inferior epi-gastric artery after the typical transversal incision. Furthermore the blood supply by the superior epi-gastric artery is also compromised by post-operative tension on the abdominal wall [11]. Although a lot of research on tissue perfusion has been performed, we still do not have a full scientific understanding of the processes of tissue perfusion, especially in specific operative settings [13-15].

Therefore, this study focussed on patients undergoing abdominal dermolipectomy after massive weight loss because the abdominal skin of these patients is even more compromised by the long-time overweight. The purpose of this study was to assess spatial and temporal patterns of abdominal wall perfusion and abdominal wall oxygen consumption in vivo with a combined laser Doppler flow metry and white light spectroscopy device, in order to identify regional differences on the one hand and pre- and post-operative changes on the other hand. In particular, we wanted to assess the horizontal and vertical perfusion zones along the resection line in order to contribute to the understanding of the high number of wound healing complications after abdominal dermolipectomy.

\section{Material and Methods}

17 consecutive randomly assigned patients (13 females, 4 males) of the Department of Plastic and Hand Surgery were included in this study. Informed consent was given by all patients prior to operation. All patients had achieved massive weight loss and were planned for an abdominal dermolipectomy. 
Dragu et al.: Evaluation of Intra-Operative Abdominal Wall Perfusion in Post-

Bariatric Abdominal Dermolipectomy

Table 1. Patient's age, gender, BMI, weight of resected tissue, nicotine consumption, preservation of umbilicus

\begin{tabular}{llllll}
\hline Age, years & Gender & BMI, kg/m & $\begin{array}{l}\text { Nicotine } \\
\text { consumption }\end{array}$ & $\begin{array}{l}\text { Weight of resected } \\
\text { tissue, }\end{array}$ & $\begin{array}{l}\text { Umbilicus } \\
\text { preserved }\end{array}$ \\
\hline 33 & f & 24.4 & no & 1,700 & yes \\
25 & m & 27.2 & n/s & 1,400 & yes \\
67 & f & 24.5 & no & 850 & yes \\
50 & f & 31.6 & n/s & 3,100 & yes \\
50 & f & 29.4 & n/s & 1,763 & yes \\
53 & f & 26.9 & no & 1,511 & yes \\
50 & f & 25.9 & n/s & 800 & yes \\
44 & f & 33.2 & yes & 3,252 & yes \\
37 & m & 34.6 & n/s & 3,300 & yes \\
28 & f & 26.9 & yes & 2,207 & yes \\
20 & m & 27.7 & n/s & 1,100 & yes \\
27 & f & 31.8 & no & 3,970 & yes \\
27 & f & 32 & no & 2,290 & yes \\
58 & f & 24.5 & yes & 1,400 & yes \\
48 & m & 36.6 & no & 4,000 & yes \\
39 & f & 27.8 & no & 2,100 & yes \\
40 & f & 30.8 & n/s & 1,100 & \\
\hline
\end{tabular}

Surgery was performed in a standardised manner with a horizontal caudal incision line and cranial mobilisation up to the xyphoid, with circumcision and transposition of the umbilicus, without plication of the rectus sheath and with final subcutaneous suturing of scarpa's fascia. Patient data and characteristics are shown in table 1 . The average age was $41 \pm 13$ years, and the average BMI was $29.2 \pm 3.7 \mathrm{~kg} / \mathrm{m}^{2}$ after massive weight loss and due to bariatric surgery. The weight of the resected tissue was 2,108 $\pm 1,054 \mathrm{~g}$. Surgery was performed in all cases in less than $150 \mathrm{~min}$ by two experienced surgeons who had the same level of knowledge, using the same technique and materials. The average intra-operative blood loss in all patients was less than $250 \mathrm{ml}$ as documented in the operative protocols.

The perfusion of the abdominal wall was accessed by applying the tissue spectrophotometer 02C (Lea Medizintechnik GmbH, Gießen, Germany). This device allows determining the tissue oxygen supply by combining white light spectroscopy and laser Doppler technique. White light spectroscopy detects the oxygen saturation of haemoglobin $\left(\mathrm{SO}_{2}\right)$ and the relative amount of haemoglobin in tissues (rHb), specifically in the post-capillary system. The laser Doppler technique provides information about the parameter blood flow (BF) in micro-circulation [16]. Tissue perfusion was assessed by monitoring the above mentioned parameters $\left(\mathrm{SO}_{2}, \mathrm{rHB}\right.$ and $\left.\mathrm{BF}\right)$ at three (I-III) defined time points of the operation. The first measurement started before the first incision (I). After starting the surgery and mobilising the abdominal wall up to the costal arch, the second measurement was performed (II). Finally, abdominal wall perfusion was assessed after tissue resection was completed and the sub-cuticular sutures were finished (III). In every patient and at each of the three measuring points two different measurement protocols were applied. Both protocols were subsequently conducted. Protocol A consists of six measuring points (M1-6) in median line (fig. 1A) in order to investigate the perfusion gradient in craniocaudal direction. Measuring point 1 (M1) was located right below the xiphoid process, M2 in the upper adominal region, M3 supraumbilical, M4 in the zone of the future cranial wound margin, M5 in the resected tissue part and M6 in the pubic region (caudal wound margin). In protocol B seven measuring points (M1-7) were applied (fig. 1B) to assess perfusion along the resection line. M1 was located in the median line at the cranial wound margin, M2 in the resected tissue part, M3 in the left lateral cranial wound margin, M4 in the right lateral cranial wound margin, M5 in median pubic region (caudal wound margin), M6 in the left hip region and M7 in the right hip region. Therefore, M1, M3 and M4 represent the cranial wound margin, and M5, M6 and M7 represent the caudal wound margin. As M5 in protocol A and M2 in protocol B are located in the area that was resected, for these two points only measurements I and II exist. 


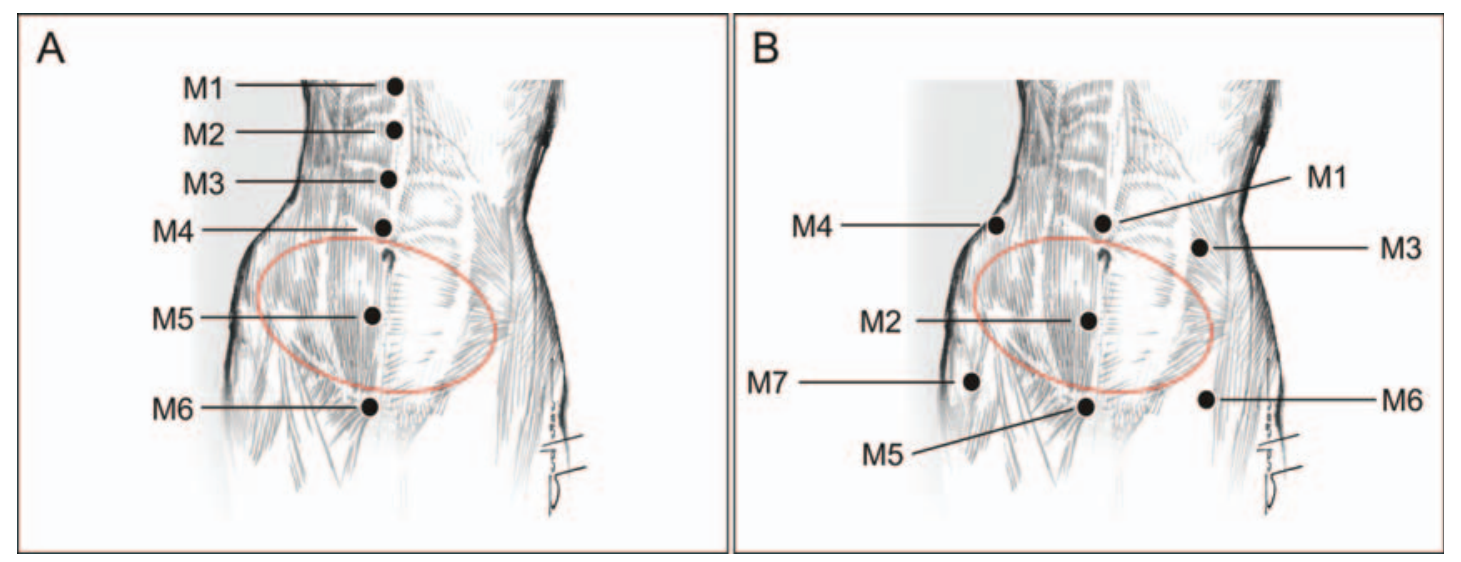

Fig. 1. A Distribution of measuring points of protocol A. B Distribution of measuring points of protocol B.

All obtained data are given as a mean \pm standard deviation (SD). The Kolgomorov-Smirnov test was used to test for Gaussian distribution, which showed a normal distribution. Therefore, a paired Student's t-test was applied to test for statistic significances, when comparing tissue perfusion of time point I to II and time point I to III. Statistical significance was defined for $\mathrm{p}<0.05$, high significance for $\mathrm{p}<0.01$ and very high significance for $\mathrm{p}<0.001$.

\section{Results}

The results of measurement protocol A and B are illustrated in fig. 2 and fig. 3. The alteration of $\mathrm{SO}_{2}$ in measurement protocol $\mathrm{A}$ is depicted in figure 2. At the beginning of the surgery, oxygen saturation declined from $\mathrm{M} 1\left(68 \% \mathrm{SO}_{2}\right)$ to $\mathrm{M} 5\left(46 \% \mathrm{SO}_{2}\right)$, and the lowest $\mathrm{SO}_{2}$ was registered at M5; at $\mathrm{M} 6$ in the mons pubis area $\mathrm{SO}_{2}$ was $58 \%$ ). After mobilisation, $\mathrm{SO}_{2}$ even significantly decreased at M5 to $26 \% \mathrm{SO}_{2}$. After the resection of the tissue, the lowest $\mathrm{SO}_{2}$ that had significantly declined was registered at $\mathrm{M} 4$ with $33 \% \mathrm{SO}_{2}$, which at that time represented the cranial median wound edge. In the most caudal point $\mathrm{M} 6, \mathrm{SO}_{2}$ showed a very highly significant increase after mobilisation $\left(70 \% \mathrm{SO}_{2}\right)$ and resection $\left(70 \% \mathrm{SO}_{2}\right)$. The rHB in tissue is shown in figure 2. At the start of the surgery the highest rHB is measured in the most caudal measuring point M6. After mobilisation, rHB showed very highly significant increases at M4-M6 $+23.7 \%$, $37.1 \%, 19.1 \%)$ and is still very highly significantly elevated after the surgery at M3 $(+4.7 \%)$, M4 (+15.8\%) and M6 (+14.9\%). The highest BF before the surgery, as depicted in figure 2, was measured at M6. After mobilisation, BF increased highly significantly at M2, and after surgery the BF remained highly significantly increased at the most caudal point M6.

The results of measuring protocol $\mathrm{B}$ are shown in figure 3 . Figure $3 \mathrm{~A}$ shows the alteration of micro-perfusion along the cranial wound margin, and figure 3B illustrates micro-perfusion along the caudal wound margin. As depicted in figure $3, \mathrm{SO}_{2}$ significantly increased along the caudal wound margin at M5, M6 and M7 after mobilisation. After suturing $\mathrm{SO}_{2}$ remained increased at M3-M7 but declined very high significantly at M1 from 59\% to 39\% (-33.9\%). rHBhighly significantly increased in all measuring points (M1-M7) after mobilisation and remained high significantly increased at M1, M3, M4 and M5, but on a lower level compared to the time of mobilisation. Relative BF highly significantly increased after mobilisation ( $+42.3 \%)$ and after suturing $(+42 \%)$ at M5 (caudal median wound edge). At M1 (cranial median wound edge) BF highly significantly rose after mobilisation (+88.9\%) and remained on an elevated level after wound closure $(+38.9 \%)$ as compared to the pre-operative measurement. 


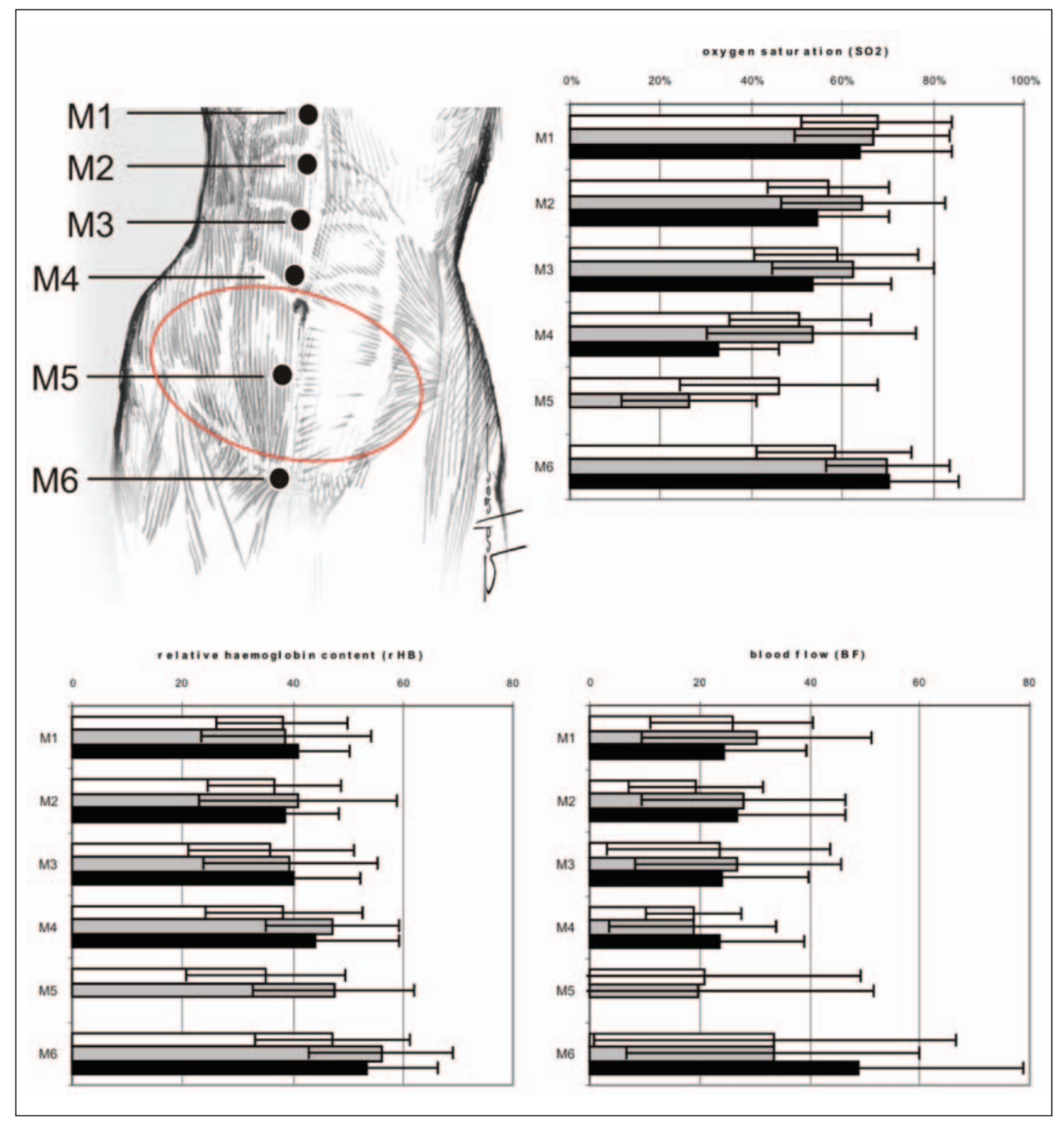

Fig. 2. Micro-perfusion parameters in cranio-caudal direction; the first table shows $\mathrm{SO}_{2}$, the second shows the rHB, and the third one shows relative BF. White bars represent measuring before surgery, grey bars represent measurements taken after mobilisation, and black bars represent measurements after resection and suturing.

\section{Discussion}

Rapid wound healing without complications is desirable in all surgical disciplines. Sufficient oxygen supply to the wound edges is believed to be essential in this context. Often micro-perfusion of wound tissue is impaired by the operation itself, as it has been shown for abdominal dermolipectomy [11]. In order to develop strategies to ameliorate microperfusion after abdominal dermolipectomy a profound understanding and inventory of micro-perfusion and especially oxygen consumption in vivo after abdominal dermoli- 


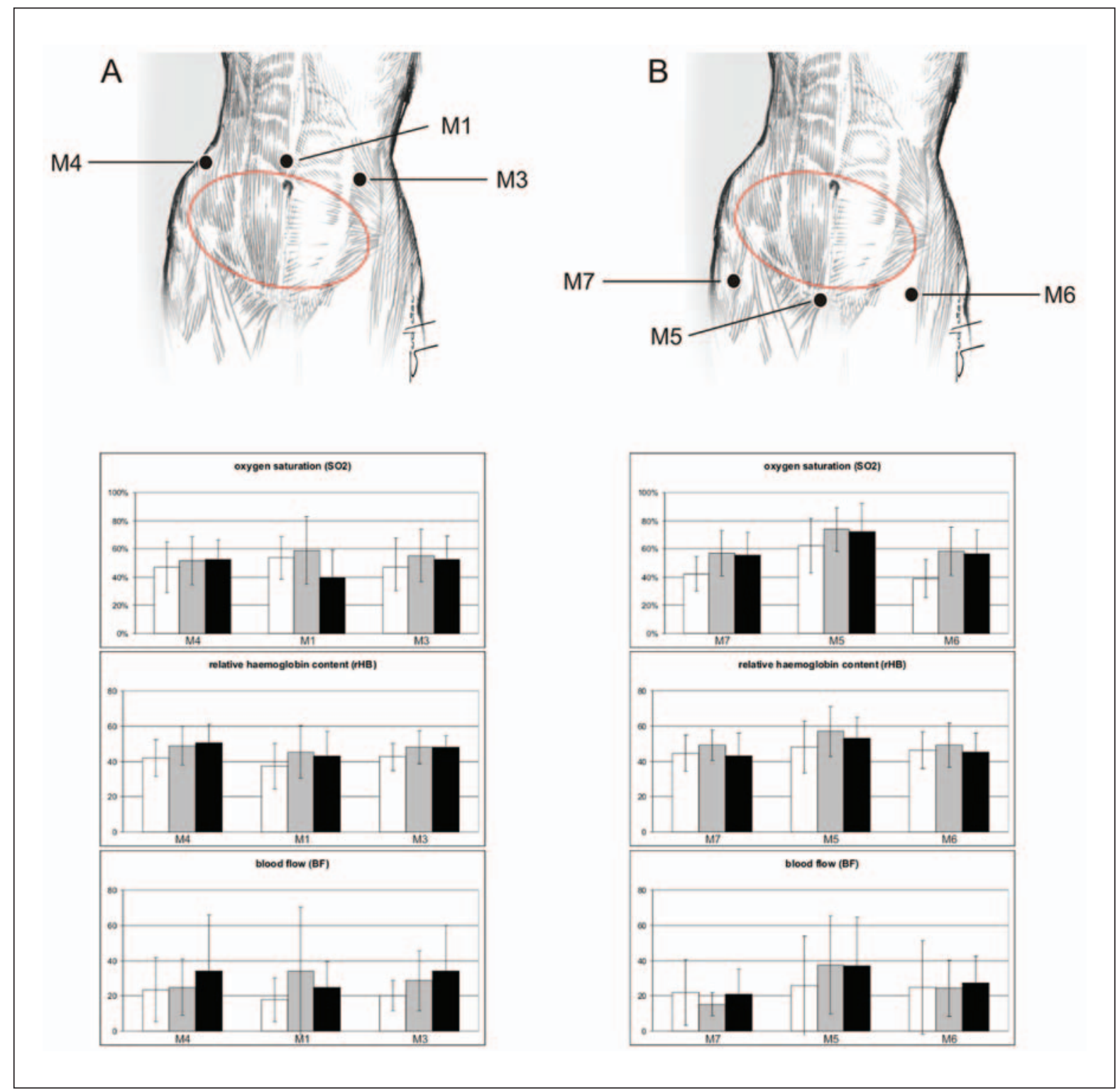

Fig. 3. Micro-perfusion parameters along the wound margin. A Perfusion along cranial wound margin. B Perfusion along caudal wound margin. The first table shows $\mathrm{SO}_{2}$, the second one shows rHB and the third one shows relative BF. White bars represent measuring before surgery, grey bars represent measurements taken after mobilisation, and black bars represent measurements after resection and suturing.

pectomy is essential. Several studies proved that the spectrophotometer 02C allows reliable non-invasive evaluation of micro-perfusion and oxygen saturation. In diabetic patients it has been demonstrated that it acts as a useful tool to identify ischaemic regions on the one hand and to distinguish foot ulcers requiring surgical intervention on the other hand [1719]. A study on skin burn injuries by Merz et al. [20] showed that the assessment of blood flow via laser Doppler technique helps to differentiate between regions of sufficient primary wound healing and regions of prolonged wound healing.

Reducing the complication rates after post-bariatric plastic surgery is certainly worth any effort. The numbers of this type of surgery have been and are still going to rise expo- 
nentially in the next years, given the momentary evolution of interventions [21], as obesity is one of the leading health problems of western civilisations $[9,22]$. Understanding the perfusion patterns of the abdominal wall during and after surgery might help to identify optimisation strategies for post-bariatric surgery, which might subsequently be transferred to other surgical topics afterwards.

To our knowledge this is the first study applying the combined laser white light spectrophotometer $02 \mathrm{C}$ in investigating the perfusion of the abdominal wall during and after abdominal dermolipectomy. Before the surgical incision, the lowest $\mathrm{SO}_{2}$ could be measured in the infra-umbilical region at M5 with 46\% (protocol A) and M2 with 37\% (protocol B) which would be resected by the surgery (fig. 1A,B). This might be explained by the fact that this part of the abdominal wall was strongly compromised by former obesity, which resulted in lax skin after massive weight loss. After the incision and mobilisation up to the costal arch, $\mathrm{SO}_{2}$ in the infra-umbilical region even decreased on average by $-33.9 \%$, as the blood supply originating from caudal arteries, based on the system from the inferior epi-gastric artery, was interrupted. This hypothesis might be supported by the fact that measurement data in the most caudal points M6 (protocol A) and M5 (protocol B), which are located in the pubic region, showed a significantly increased $\mathrm{SO}_{2}$ (on average of $+20.2 \%$ ) (fig. $1 \mathrm{~A}, \mathrm{~B}$ ). After resection of the abdominal flap and suturing, a significant decrease of $\mathrm{SO}_{2}$ (on average $-30.9 \%$ ) was measured in the former para-umbilical region M4 (protocol A) and M1 (protocol $B$ ) which from that time on represented the most cranial median part of the wound edge (fig. $1 \mathrm{~A}, \mathrm{~B}$ ). One would have expected that $\mathrm{SO}_{2}$ would at least remain stable in this region as a large amount of tissue needing blood supply from the same vessels was removed. The decreased $\mathrm{SO}_{2}$ might be due to impaired blood supply caused by post-operative tension on parts of the abdominal wall. Compared to the pubic region and the caudal wound edge region (M7, M5 and M6) (fig. 1B), where BF significantly increased post-operatively, the BF remained on a low level in all cranial wound edge regions (fig. 1B). The significantly increasing haemoglobin content measured in all regions (M1-M6 in protocol A) could indicate a slight venous stasis in this area as this parameter represents the effective amount of blood in the capillary system (fig. 1A).

Results of measuring protocol B, which was focussed on micro-perfusion and oxygen consumption along the horizontal resection line, show that $\mathrm{SO}_{2}$ was highly significantly increased after mobilisation of the tissue and after suturing in the caudal parts of the wound margin (fig. 3B). Interestingly, the highest $\mathrm{SO}_{2}$ was measured in the median line $(73.8 \%$ (II); $72.3 \%$ (III)) and decreased towards the lateral wound corners (left corner: $56.9 \%$ (II), $55.7 \%$ (III); right corner: 58.2\% (II), 56.7\% (III)). Along the cranial wound edge margin (fig. 3A), $\mathrm{SO}_{2}$ at first increased after mobilisation, but after suturing it significantly declined in the median line (53.7\% (I) vs. 39.5\% (III)). Summing up, these results indicate that abdominal dermolipectomy not only reduces superfluous skin and fat in the lower abdomen, but also highly elevates $\mathrm{SO}_{2}$ along the horizontal resection line, except in the cranial medial wound edge region. $\mathrm{rHB}$ and $\mathrm{BF}$ very highly significantly increased along the caudal wound margin (fig. 3B) after mobilisation and suturing with a peak in the median line. Along the cranial wound margin, the $\mathrm{rHB}$ also increased, and, correlating with the $\mathrm{SO}_{2}$, the lowest $\mathrm{rHB}$ was measured along the median line. Interestingly, BF (fig. $3 \mathrm{~A}$ ) along the cranial wound margin rose after mobilisation, but declined in median line after suturing (34.1 (II) vs. 24.8 (III)). The elevated blood flow in this region after mobilisation might be attributed to the delay phenomenon. It is based on the fact that the size of blood vessels in a certain region increases after other nutritive vessels of this region had been dissected [23, 24]. These results correlate with the study of Mayr et al., [11] who found significantly decreased perfusion post-operatively in the zone forming the cranial wound margin by using indocyanine green perfusography. 
The results are indicative that the medial cranial wound margin maybe predisposed for wound healing complications, as micro-perfusion of this area was compromised after the operation. Nevertheless, the average measured $\mathrm{SO}_{2}$ of $39.5 \%$ (fig. 1) was only marginally below the limit of $40 \%$, where most experts would assume the beginning of tissue hypoxia [25]. Thus, this study shows on the one hand that surgery compromised micro-perfusion of the wound margin, but that on the other hand the abdominal region seems to have enough reserves to assure micro-perfusion in massive weight loss patients when respecting specific micro-perfusion patterns. Regarding the perfusion characteristics of the abdominal wall except for the median cranial wound margin - it is noticeable that micro-perfusion, especially in caudal regions below the incision line in the pubic area, changed for the better after surgery (fig. 3B). This study corroborates the clinical impression that wound healing is mostly altered in the medial part of the mobilised abdominal skin flap since it shows a distinct alteration of the micro-perfusion pattern during abdominoplasty that correlates with this clinically known phenomenon. Nevertheless, to further validate these findings, we believe that more randomised clinical trials including different patients with different operation techniques should be carried out in the future to gain more experience on the specific micro-perfusion patterns of mobilised tissues after dermolipectomies. Finally, these data may support the clinical practice to place suture tension more on the lateral parts of the wound edges, and not on the median parts as advocated with the high lateral tension abdominoplasty by Rosenfield and Lockwood [26, 27]. In the light of high post-operative complications after post-bariatric plastic surgery, our data may help to reduce these complication rates due to a better understanding of the micro-perfusion patterns of the abdominal wall during post-bariatric abdominal dermolipectomy. These data underline the necessity of critically assessing the amount of tissue that should be resected by abdominal dermolipectomy as the perfusion is not only impaired by dissecting nutritive vessels but also by post-operative tension on the abdominal wall.

\section{Disclosure Statement}

All contributing authors declare that they have no conflicts of interest.

\section{References}

1 Kelly HA: Excision of fat of the abdominal wall lipectomy. Surg Gynecol Obstet 1910;10:229.

- 2 Dragu A, Schnürer S, Unglaub F, Wolf MB, Beier JP, Kneser U, Horch RE: Wide topical negative pressure wound dressing treatment for patients undergoing abdominal dermolipectomy following massive weight loss. Obes Surg 2011;21:1781-1786.

3 Dragu A, Unglaub F, Wolf MB, Beier JP, Schnabl SM, Kneser U, Leffler M, Horch RE: Scars and perforatorbased flaps in the abdominal region: a contraindication? Can J Surg 2010;53:137-142.

4 Grazer FM: Abdominoplasty. Plast Reconstr Surg 1973;51:617-623.

- 5 Dragu A, Birkholz T, Kleinmann JA, Schnürer S, Munch F, Cesnjevar R, Schmidt J, Taeger C, Kneser U, Horch RE: Extracorporeal perfusion of free muscle flaps in a porcine model using a miniaturized perfusion system. Arch Orthop Trauma Surg 2011;131:849-855.

6 ASAPS: 2009 Cosmetic surgery national data bank statistics www.surgery.org/sites/default/files/2009stats.pdf (last accessed September 25, 2012).

7 van der Beek ES, van der Molen AM, van Ramshorst B: Complications after body contouring surgery in postbariatric patients: the importance of a stable weight close to normal. Obes Facts 2011;4:61-66.

- 8 Neaman KC,Hansen JE: Analysis of complications from abdominoplasty: a review of 206 cases ata university hospital. Ann Plast Surg 2007;58:292-298.

- 9 Wang Y, Beydoun MA: The obesity epidemic in the United States - gender, age, socioeconomic, racial/ethnic, and geographic characteristics: a systematic review and meta-regression analysis. Epidemiol Rev 2007;29: $6-28$. 
10 Agha-Mohammadi S, Hurwitz DJ: Enhanced recovery after body-contouring surgery: reducing surgical complication rates by optimizing nutrition. Aesthetic Plast Surg 2010;34:617-625.

-11 Mayr M, Holm C, Hofter E, Becker A, Pfeiffer U, Muhlbauer W: Effects of aesthetic abdominoplasty on abdominal wall perfusion: a quantitative evaluation. Plast Reconstr Surg 2004;114:1586-1594.

12 Huger WE Jr: The anatomic rationale for abdominal lipectomy. Am Surg 1979;45:612-617.

-13 Arkudas A, Beier JP, Pryymachuk G, Hoereth T, Bleiziffer O, Polykandriotis E, Hess A, Gulle H, Horch RE, Kneser U: Automatic quantitative micro-computed tomography evaluation of angiogenesis in an axially vascularized tissue-engineered bone construct. Tissue Eng Part C Methods 2010;16:1503-1514.

-14 Bleiziffer O, Hammon M, Naschberger E, Lipnik K, Arkudas A, Rath S, Pryymachuk G, Beier JP, Sturzl M, Horch RE, Kneser U: Endothelial progenitor cells are integrated in newly formed capillaries and alter adjacent fibrovascular tissue after subcutaneous implantation in a fibrin matrix. J Cell Mol Med 2011;15: 2452-2461.

15 Dragu A, Schnürer S, Surmann-Schmitt C, von der Mark K, Sturzl M, Unglaub F, Wolf MB, Leffler M, Beier JP, Kneser U, Horch RE: Gene expression analysis of ischemia and reperfusion in human microsurgical free muscle tissue transfer. J Cell Mol Med 2011;15:983-993.

16 Krug A: 02C (oxygen to see). 2007. www.lea.de/pdf/Lea/product/050614_Method_deu.pdf (last accessed September 25, 2012).

17 Rajbhandari SM, Harris ND, Tesfaye S, Ward JD: Early identification of diabetic foot ulcers that may require intervention using the micro lightguide spectrophotometer. Diabetes Care 1999;22:1292-1295.

18 Beckert S, Witte MB, Konigsrainer A, Coerper S: The impact of the micro-lightguide 02C for the quantification of tissue ischemia in diabetic foot ulcers. Diabetes Care 2004;27:2863-2867.

19 Forst T, Hohberg C, Tarakci E, Forst S, Kann P, Pfutzner A: Reliability of lightguide spectrophotometry (O2C) for the investigation of skin tissue microvascular blood flow and tissue oxygen supply in diabetic and nondiabetic subjects. J Diabetes Sci Technol 2008;2:1151-1156.

20 Merz KM, Pfau M, Blumenstock G, Tenenhaus M, Schaller HE, Rennekampff HO: Cutaneous microcirculatory assessment of the burn wound is associated with depth of injury and predicts healing time. Burns 2010;36: 477-482.

21 Matarasso A, Roslin MS, Kurian M: Bariatric surgery: an overview of obesity surgery. Plast Reconstr Surg 2007;119:1357-1362.

-22 Naal FD, Neuerburg C, Salzmann GM, Kriner M, von Knoch F, Preiss S, Drobny T, Munzinger U: Association of body mass index and clinical outcome 2 years after unicompartmental knee arthroplasty. Arch Orthop Trauma Surg 2009;129:463-468.

23 Ozkan O, Coskunfirat OK, Ozgentas HE, Yildirim I, Dikici MB: Is it possible to increase the survival of the transverse rectus abdominis musculocutaneous flap following previous abdominoplasty using a delay procedure? An experimental study in the rat. Plast Reconstr Surg 2005;116:1945-1952.

24 Myers MB, Cherry G: Mechanism of the delay phenomenon. Plast Reconstr Surg 1969;44:52-57.

-25 Fife CE, Smart DR, Sheffield PJ, Hopf HW, Hawkins G, Clarke D: Transcutaneous oximetry in clinical practice: consensus statements from an expert panel based on evidence. Undersea Hyperb Med 2009;36:43-53.

-26 Lockwood T: High-lateral-tension abdominoplasty with superficial fascial system suspension. Plast Reconstr Surg 1995;96:603-615.

27 Rosenfield LK: High tension abdominoplasty 2.0. Clin Plast Surg 2010;37:441-465. 